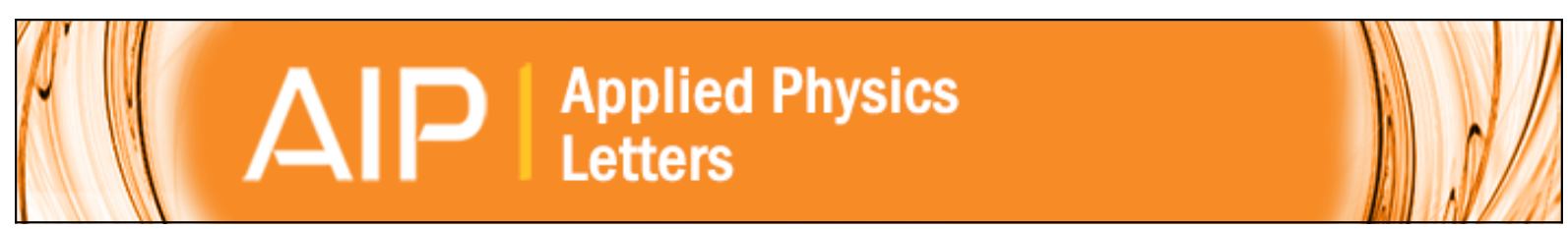

\title{
Seebeck coefficient in silicon nanowire arrays
}

Emiljana Krali and Zahid A. K. Durrani

Citation: Applied Physics Letters 102, 143102 (2013); doi: 10.1063/1.4800778

View online: http://dx.doi.org/10.1063/1.4800778

View Table of Contents: http://scitation.aip.org/content/aip/journal/apl/102/14?ver=pdfcov

Published by the AIP Publishing

\section{Advertisement:}

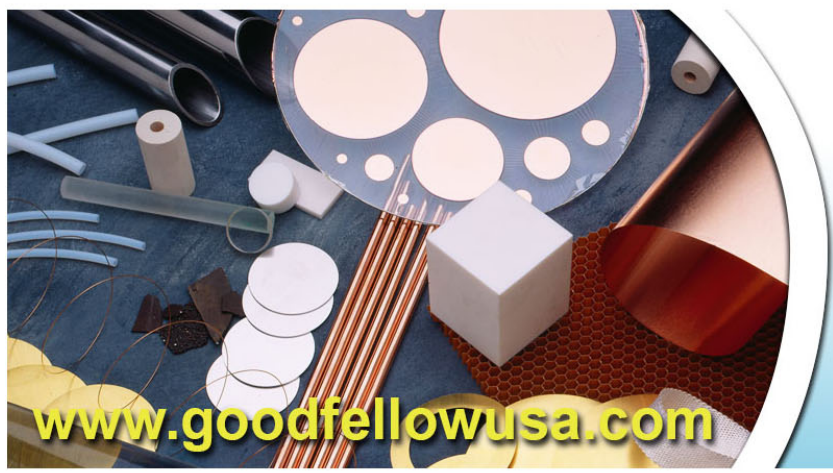

BOAdFEl/OU

metals • ceramics $\cdot$ polymers composites • compounds • glasses

Save $5 \% \cdot$ Buy online 70,000 products $\cdot$ Fast shipping 


\title{
Seebeck coefficient in silicon nanowire arrays
}

\author{
Emiljana Krali and Zahid A. K. Durrani ${ }^{\text {a) }}$ \\ Department of Electrical and Electronic Engineering, Imperial College London, South Kensington Campus, \\ London SW7 2AZ, United Kingdom
}

(Received 15 October 2012; accepted 25 March 2013; published online 8 April 2013)

\begin{abstract}
We measure the Seebeck coefficient $S$ in large arrays of lightly doped $n$-Si nanowires (SiNWs). Our samples consist of $\sim 10^{7} \mathrm{NWs}$ in parallel, forming a "bulk" nano-structured material. We find that the phonon drag component of $S$, a manifestation of electron-phonon scattering in the sample, is heavily suppressed due to surface scattering, and that there is a "universal" temperature dependence for $S$. Furthermore, at room temperature, $S$ is enhanced in the arrays by up to $\sim 3$ times in comparison to bulk Si. (c) 2013 American Institute of Physics. [http://dx.doi.org/10.1063/1.4800778]
\end{abstract}

In recent years, there has been great interest in thermoelectric (TE) effects ${ }^{1-4}$ for application in alternative "clean" energy sources and for more efficient utilisation of energy. TE materials, where a temperature difference $\Delta T$ is converted into an electric potential difference $\Delta V$, are quantified using the dimensionless figure-of-merit ${ }^{5} Z T=S^{2} \sigma T / \kappa$, where $S=\Delta V / \Delta T, \sigma$, and $\kappa$ are the Seebeck coefficient, electrical and thermal conductivity, respectively, at temperature $T$. A value of $Z T \sim 1$ is necessary for practical applications. ${ }^{6}$ While conventional bulk TE materials such as $\mathrm{Bi}_{2} \mathrm{Te}_{3}$ require a compromise between $S, \sigma$, and $\kappa$, limiting $\mathrm{ZT} \sim 1$, in nanostructured materials these parameters may be varied quasi-independently ${ }^{3,4,7,8}$ such that $Z T>1$. Here, $S$ can be increased by quantum confinement of electrons without excessively affecting $\sigma$ and furthermore, $\kappa$ may be reduced independently of the other parameters by either increased surface scattering of phonons or modification of the density-of-states. ${ }^{7,9}$ As these effects depend primarily on the length scale, there is greater freedom in material choice, and TE devices in materials even with nominally poor $Z T$ become feasible.

Bulk Si, the most widely used semiconductor and the basis of large-scale integrated circuits, has a poor $Z T \sim 0.01$, due to high thermal conductivity, ${ }^{10} \kappa=150 \mathrm{~W} / \mathrm{mK}$ at $300 \mathrm{~K}$. However, measurements on a single ${ }^{3} \mathrm{Si}$ nanowire (SiNW) and $\sim 100$ SiNWs in parallel ${ }^{4}$ have reported $Z T \sim 1$. Here, the NWs were defined in heavily doped $\left(10^{19}-10^{20} / \mathrm{cm}^{3}\right)$ material to maximise $\sigma$, and $\kappa$ was strongly reduced $\sim 1 \mathrm{~W} / \mathrm{mK}$, either due to increased surface scattering ${ }^{3}$ or thermoelastic effects. ${ }^{4}$ These observations demonstrate the potential for Si TE devices, raising the possibility of increased functionality in $\mathrm{Si}$, and direct integration of TE and electronic devices for energy scavenging.

In this paper, we measure the temperature dependence of $S$ in samples consisting of $\sim 10^{7}$ vertically aligned SiNWs (Fig. 1) fabricated using metal-assisted chemical etching (MACE) ${ }^{11,12}$ The process creates SiNWs from $\sim 30$ to $400 \mathrm{~nm}$ in diameter, with large aspect ratio up to 1:3000. We use a transient temperature and voltage measurement technique, allowing direct measurement of $S$ in thin (1 mm thickness) samples. We also use lightly doped $\left(\sim 10^{15} / \mathrm{cm}^{3}\right) n$-type Si to reduce impurity scattering of electrons and phonons. At low doping

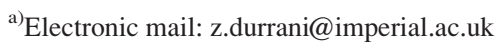

levels, phonon drag is the dominant contribution to $S$ at moderate to low temperatures ${ }^{10}$ ( 200-30 K). Unlike heavily doped SiNWs used in previous work, ${ }^{3,4}$ our low doping levels disentangle impurity scattering from surface scattering and leave the NW dimensions as the main source of scattering.

The SiNWs (Fig. 1) were synthesised from a lightly doped $n$-type silicon (100) wafer (resistivity $\rho \sim 1-5 \Omega \mathrm{cm}$, phosphorous doping from $5 \times 10^{14}$ to $3 \times 10^{15} / \mathrm{cm}^{3}$ ) using a two-step chemical etching process. ${ }^{13,14}$ Here, an electroless deposition (galvanic exchange) process deposits $\mathrm{Ag}$ nanoparticles from $\mathrm{HF} / \mathrm{AgNO}_{3} / \mathrm{H}_{2} \mathrm{O}$ solution on the $\mathrm{Si}$ surface. The nanoparticles act as catalysts for subsequent etching of the $\mathrm{NWs}$ in $\mathrm{HF} / \mathrm{H}_{2} \mathrm{O}_{2} / \mathrm{H}_{2} \mathrm{O}$ solution. The main part of Fig. 1 shows the cross-section through a $60 \mu \mathrm{m}$ long SiNW array. The insets 1-3 show higher resolution images of three areas (circled), at $\sim 5, \sim 30$, and $\sim 60 \mu \mathrm{m}$ depth. The NWs are vertically aligned, with parallel sidewalls over the full etch depth. Inset 3 shows the base of the array, where NWs join the unetched substrate, cleaved at an angle to the NW cross-section. The NW morphology is complex, with irregular crosssection. This consists of a columnar core $\sim 100-400 \mathrm{~nm}$ in width with vertically aligned attached ribs $\sim 30-100 \mathrm{~nm}$ in width. At least some of the ribs are poorly attached or may be

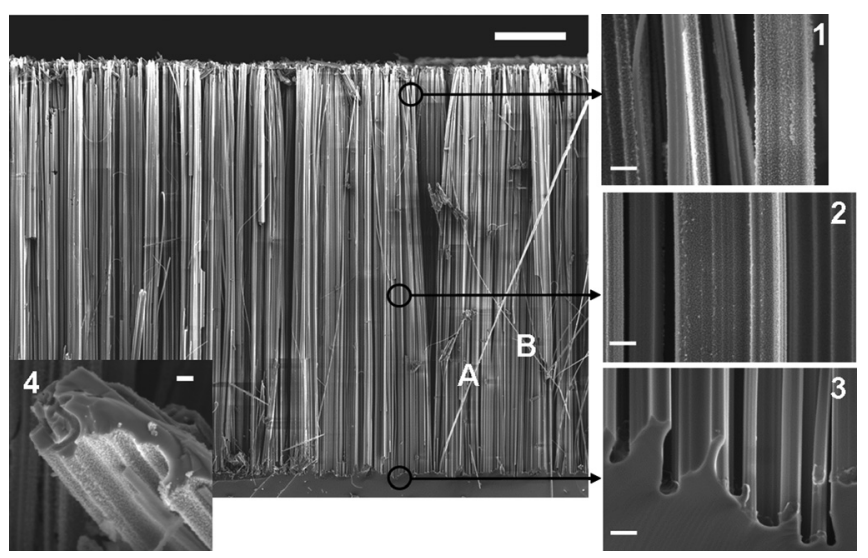

FIG. 1. Main image: Cross-sectional scanning electron micrograph through a $60 \mu \mathrm{m}$ long SiNW array, obtained using a Zeiss Ultra Plus SEM. SiNWs marked "A" and "B" are $\sim 100 \mathrm{~nm}$ and $\sim 50 \mathrm{~nm}$ wide. The scale bar is $10 \mu \mathrm{m}$. Insets 1-3 show high-resolution micrographs of the corresponding circled regions. The scale bar is $200 \mathrm{~nm}$. Inset 4 shows a broken-off section from the top of the array. The scale bar is $100 \mathrm{~nm}$. 
pinched-off, e.g., the NWs marked "A" (diameter $\sim 100 \mathrm{~nm}$ ) and "B" (diameter $\sim 50 \mathrm{~nm}$ ) may have broken off from a wider section. In each of our $0.5 \times 0.5 \mathrm{~cm}^{2}$ samples, using an array filling factor of $\sim 30 \%$ and an average NW diameter of $\sim 250 \mathrm{~nm}$, there are $\sim 10^{7} \mathrm{NWs}$. The filling factor was extracted by measuring the change in sample weight after oxidation. Samples were oxidised at $1050{ }^{\circ} \mathrm{C}$ for several hours, completely oxidising the NWs. The change in sample weight allowed extraction of the weight of NWs. Using the $\mathrm{NW}$ weight, length, and density of $\mathrm{Si}$, it is then possible to obtain the total cross-section area through the NWs and hence, the filling factor.

Insets 1-3 allow comparison of the NW surface roughness with array depth. This reduces from $\sim 10$ to $20 \mathrm{~nm}$ near the top (inset 1), to $\sim 5 \mathrm{~nm}$ at $\sim 30 \mu \mathrm{m}$ depth (inset 2), to a negligible value at the bottom (inset 3 ). This suggests that the longer a section of NW surface remains immersed in the etchant, the greater the surface roughness due to increased surface etching. Inset 4 shows a broken-off section from the top of the array. While the NW surface roughness is $\sim 20 \mathrm{~nm}$, the core is unaffected.

$S$ is measured using a transient temperature and voltage technique, allowing characterisation of thin, large area samples. In contrast, equilibrium measurements require large separation between the hot and cold ends of the sample to allow $\Delta T$ to be maintained. ${ }^{15-17}$ In previous work, ${ }^{3,4}$ single or small numbers of SiNWs were used, such that the thermal resistance was large and $\Delta T$ could be established at equilibrium. As we characterise $\sim 10^{7} \mathrm{NWs}$ in parallel, the thermal resistance is reduced, making equilibrium measurements difficult. Figure 2(a) shows our experimental apparatus schematically. The Si sample is sandwiched between a bottom (cold) $\mathrm{Cu}$ heat sink (temperature $\mathrm{T1}$ ) and a top $\mathrm{Cu}$ "hot" reservoir (temperature $T 2$ ), allowing measurement of the temperature difference $\Delta T=T 2-T 1$. Pressure is applied to the top $\mathrm{Cu}$ block, and $\mathrm{Al}$ foil (thickness $120 \mu \mathrm{m}$ ) is used to improve the $\mathrm{Si} / \mathrm{Cu}$ thermal contacts. Reduction of the bottom $\mathrm{Cu}$ block temperature, and the time lag in the top $\mathrm{Cu}$ block reaching this temperature, allows a small transient $\Delta T$ to exist across the sample. We then measure simultaneously $\Delta T$ and $\Delta V$ (open circuit voltage), as a function of time, from the point where $T 1$ is constant. Measurements are performed from 300 to $30 \mathrm{~K}$. The temperature sensor at the top $\mathrm{Cu}$ block is placed very close $(<1 \mathrm{~mm})$ to the sample, allowing measurement of temperature near the sample/Cu interface. In addition, the time constants of the exponential decays for $\Delta V$ and $\Delta T$ are similar and very long ( $\sim 10 \mathrm{~s}$ or greater). The electron distribution can then adjust as the temperature changes, and quasi-static conditions exist in the sample. This allows the use of equilibrium equations to quantify $S$. We have also performed equilibrium measurements on the NWs at room temperature, which give similar values of $S$ to our transient measurements. Finally, we consider the suitability of the top $\mathrm{Cu}$ block as a heat reservoir. The heat capacity of $\mathrm{Cu} \sim 24 \mathrm{~J} / \mathrm{mol} \mathrm{K}$ (Ref. 18) and of $\mathrm{Si}$ is $\sim 20 \mathrm{~J} / \mathrm{mol} \mathrm{K}$ (Ref. 19). The mass of the $\mathrm{Cu}$ block is $1 \mathrm{~g}$ and of the Si sample is $0.1 \mathrm{~g}$. It then follows that the heat stored in the top $\mathrm{Cu}$ block is $\sim 9$ times greater than in the $\mathrm{Si}$ sample.

Figure 2(b) shows measurements for the bulk "parent" Si sample at $160 \mathrm{~K} . \Delta V$ and $\Delta T$ decay exponentially, with similar time constants $\sim 16$ s. $S=\Delta V / \Delta T$ is then calculated from the $\Delta V-\Delta T$ plot slope. Figure 2(b) (inset) shows linear $\Delta V-\Delta T$ plots for this sample from 280 to $80 \mathrm{~K}$. Figure 2(c) shows similar plots for an $80 \mu \mathrm{m} \mathrm{SiNW} \mathrm{sample,} \mathrm{from} 270$ to (a)
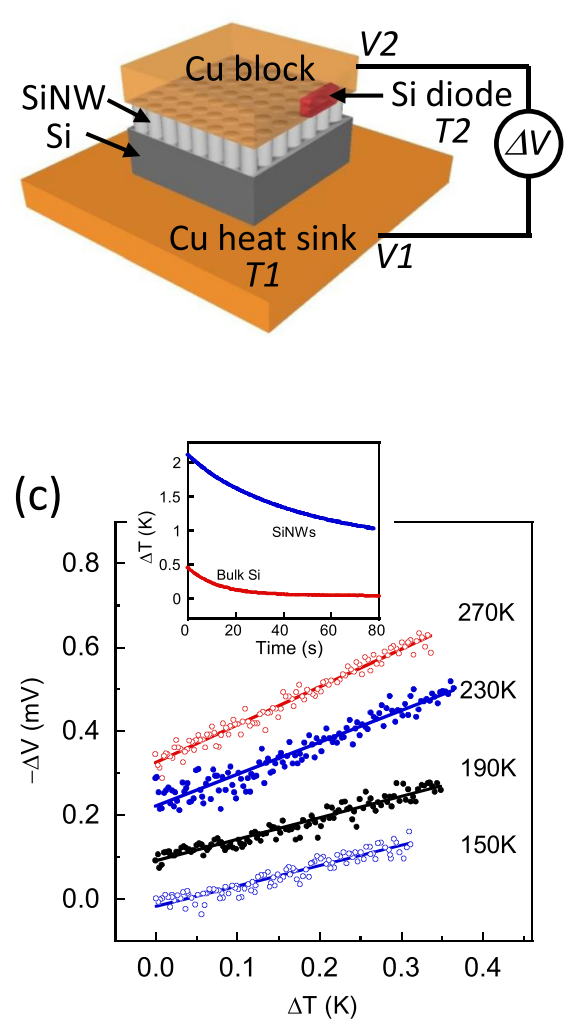

(b)

(d)
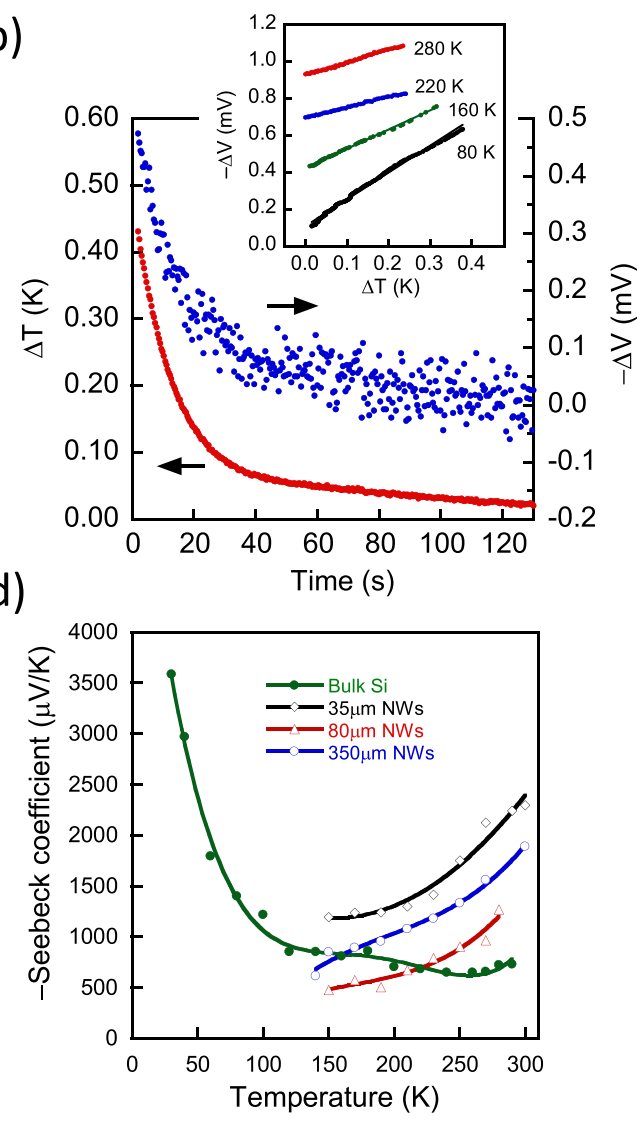

FIG. 2. (a) Schematic diagram of experimental apparatus. The bottom and top $\mathrm{Cu}$ block temperatures are $T 1$ and $T 2$, with temperature difference $\Delta T=T 2-T 1$. Voltage difference between the blocks $\Delta V$ $=V 2-V 1$. (b) $\Delta V$ and $\Delta T$ vs. time for bulk $\mathrm{Si}$, at $160 \mathrm{~K}$. Inset shows $\Delta V-\Delta T$, for $280-80 \mathrm{~K}$ heat sink temperature. Curves are offset for clarity. (c) $\Delta V-\Delta T$ for $80 \mu \mathrm{m} \mathrm{SiNWs,} \mathrm{from} 270$ to $150 \mathrm{~K}$. Curves are offset for clarity. Inset shows the exponential decay of $\Delta T$ as a function of time, for bulk Si and SiNW samples at 160 K. (d) Seebeck coefficient for bulk $\mathrm{Si}$ and for SiNW arrays with 35,80 , and $350 \mu \mathrm{m} \mathrm{NW}$ length. The solid lines are a polynomial fits to provide a guide to the eye. 
$150 \mathrm{~K}$. The inset shows $\Delta T$ as a function of time for bulk and SiNW samples at $160 \mathrm{~K}$. Figure 2(d) shows $S$ for bulk Si $\left(S_{\text {bulk }}\right)$ and for SiNW arrays $\left(S_{N W}\right)$ with 35,80 , and $350 \mu \mathrm{m}$ NW lengths. Measurements were from 290 to $30 \mathrm{~K}$ for bulk Si and 300-140 K for SiNWs.

In Figure 2(d), $S_{N W}$ is calculated as follows. We define the equations

$$
\begin{gathered}
\frac{\Delta T_{N W}}{\Delta T}=\frac{1}{1+\Delta T_{\text {bulk }} / \Delta T_{N W}}, \\
\Delta T_{\text {bulk }} \propto \frac{1}{\kappa_{B}} \frac{L_{B}}{A_{B}} ; \quad \Delta T_{N W} \propto \frac{1}{\kappa_{N W}} \frac{L_{N W}}{F \times A_{B}}, \\
\frac{\Delta V_{N W}}{\Delta V}=\frac{1}{1+\Delta V_{\text {bulk }} / \Delta V_{N W}}=\frac{1}{1+S_{\text {bulk }} \Delta T_{\text {bulk }} / S_{N W} \Delta V_{N W}},
\end{gathered}
$$

where $\left(\Delta V_{N W}, \Delta T_{N W}\right)$ and $\left(\Delta V_{b u l k}, \Delta T_{b u l k}\right)$ are the voltage and temperature across the SiNWs and the bulk Si substrate, respectively. Furthermore, $\left(\kappa_{B}, L_{B}\right)$ and $\left(\kappa_{N W}, L_{N W}\right)$ are the thermal conductivity and length in the bulk Si and SiNWs, respectively, $A_{B}$ is the bulk Si surface area, and $F$ is the filling factor of the SiNW array. In our case, $F=30 \%$, while for $\kappa_{N W}$ and $\kappa_{B}$, we use data from literature. ${ }^{3,10}$ Here, $\kappa_{N W}$ is used only to estimate $\Delta T$ across the SiNW and the bulk substrate. In principle, a transient method allows direct measurement of $\kappa_{N W}$. However, this would require an estimate of the heat loss from the top $\mathrm{Cu}$ block, e.g., through the leads connecting to the block and the temperature sensor. The time constants for bulk $\left(\tau_{B}\right)$ and the SiNWs $\left(\tau_{S i N W}\right)$ are very different, with $\tau_{B}=16.8$ and $\tau_{S i N W}=44.12$ (Fig. 2(c) inset). The ratio of the time constants $\tau_{B} / \tau_{S i N W} \propto \kappa_{S i} / \kappa_{N W}$ suggests that $\kappa_{N W}$ is reduced to $\sim 0.38 \kappa_{S}$, a value within the range reported for SiNWs also fabricated by a MACE process. ${ }^{3}$ Substituting Eq. (3) into the ratio $S_{N W} / S=\left(\Delta V_{N W} / \Delta V\right) /$ $\left(\Delta T_{N W} / \Delta T\right)$, where $S$ is the Seebeck coefficient for the entire SiNW/Si bulk sample, and solving for $S_{N W}$, we have

$$
S_{N W}=S\left(1-\frac{S_{\text {bulk }}}{S}\right) \frac{\Delta T}{\Delta T_{N W}}+\frac{S_{\text {bulk }}}{S} .
$$

Here, $\Delta T / \Delta T_{N W}$ is given by Eqs. (1) and (2), and $S_{b u l k}$ and $S$ are given by our measured data. It was not possible to measure the SiNWs below $\sim 140 \mathrm{~K}$, due to increasing sample resistance. At room temperature, $S_{N W}>S_{\text {bulk }}$ by up to $\sim 3$ times. However, $S_{\text {bulk }}$ increases and $S_{N W}$ decreases with decreasing $T$ and at lower $T, S_{N W}<S_{\text {bulk }}$ (e.g., at $220 \mathrm{~K}$ for the $80 \mu \mathrm{m}$ sample).

The increase in $S_{b u l k}$ is caused by strong enhancement of phonon drag. ${ }^{10}$ The decrease in $S_{N W}$ then implies suppression of phonon drag. Typically, $S_{\text {bulk }}=S_{p}+S_{d}$, where $S_{p}$ is the phonon drag and $S_{d}$ is the electron diffusion component. In phonon drag, momentum transfer from phonons to electrons via electron-phonon scattering increases the number of electrons reaching the cold side and therefore $S . S_{p}$ is large in lightly doped in comparison with heavily doped materials ${ }^{10}$ as in the later case, impurity scattering of phonons suppresses $S_{p}$.

In lightly doped bulk semiconductors, $S_{d}$ is given by ${ }^{20,21}$

$$
S_{d}=-\frac{k_{B}}{e}\left(\frac{E_{C}-E_{F}}{k_{B} T}+\left(r+\frac{5}{2}\right)\right)
$$

Here, $k_{B}$ is the Boltzmann constant, $E_{F}$ is the Fermi energy, $E_{C}$ is the conduction band energy, $e$ is the electron charge, and $r$ is a scattering factor, assumed to be -0.5 if phonon scattering of carriers dominates over impurity scattering. ${ }^{21}$ $E_{F}$ can be calculated using 22

$n_{d}\left(1-\left(\frac{1}{2} \exp \left(\frac{E_{D}-E_{F}}{k_{B} T}\right)+1\right)^{-1}\right)=n_{0} \exp \left(\frac{E_{F}-E_{C}}{k_{B} T}\right)$.

Here $n_{d}$ is the ionised donor concentration, $E_{D}=0.045 \mathrm{eV}$ is the donor (phosphorous) energy, $n_{0}=2\left(2 \pi m^{*} k_{B} T / h^{2}\right)^{3 / 2}$ is the conduction band effective density of states, $m^{*}=1.08$ $\times$ electron rest mass is the electron effective mass, and $h$ is Planck's constant. Finally, $S_{p}$ is given by ${ }^{20}$

$$
S_{p}=-\frac{\beta v_{p} l_{p}}{\mu_{e} T}
$$

Here, $v_{p}$ and $l_{p}$ are the velocity and mean free path of phonons participating in phonon drag, $\mu_{e}$ is the electron mobility, and $\beta$ characterises electron-phonon interaction. At room temperature and above, phonon-phonon interaction (Umklapp scattering) is predominant and $\beta \approx 0$. This implies that $S_{p}$ may be neglected at room temperature and $S_{\text {bulk }} \approx S_{d}$. As the temperature is lowered, Umklapp scattering becomes increasingly difficult, leading to $\beta>0$. At low temperatures, ${ }^{21}$ phonon-phonon interaction is almost void and $\beta \approx 1$. Furthermore, as $\mu_{e} \propto T^{-3 / 2}$ in lightly doped $\mathrm{Si}$ and $v_{p}$ may be assumed to be constant, we have $S_{p} T^{-1 / 2} \propto l_{p}$, providing a means to obtain the temperature dependence of $l_{p}$ from $S_{p}$.

Figure 3(a) shows $S_{\text {bulk }}$ and $S_{d}$ calculated using Eqs. (1) and (2) for $10^{15} / \mathrm{cm}^{3}$ doping concentration, where $S_{\text {bulk }} \approx S_{d}$ $\approx 730 \mu \mathrm{V} / \mathrm{K}$ at $290 \mathrm{~K}$. $S_{\text {bulk }}$ is, however, slightly smaller than $S_{d}$ from $220 \mathrm{~K}<T<300 \mathrm{~K}$, as we may have overestimated $S_{d}$ if $r<-0.5$ or underestimated $S_{\text {bulk }}$ due to unaccounted interface temperature drops. $S_{p}=S_{b u l k}-S_{d}$ is extracted assuming that $S_{p}$ is negligible at room temperature. $S_{p}$ increases below $\sim 250 \mathrm{~K}$ and $\sim 80 \mathrm{~K}, S_{p}>S_{d}$ (e.g., at $\left.30 \mathrm{~K}, S_{p}=0.7 S_{\text {bulk }}\right)$. The temperature dependence of $l_{p}$ is found by plotting $S_{p} T^{-1 / 2} \propto l_{p}$ vs. $T$ on a log-log scale (Fig. 3(a) inset). We find a power-law dependence, with $l_{p} \propto T^{n}$ where $n=-2.3$. This is associated with the reduction in Umklapp scattering of phonons with reducing $T$ and is similar to published data, ${ }^{10}$ where $n \sim 2.1$. Finally, Fig. 3(b) shows $S_{N W}$, normalized to room-temperature values, vs. $T$. All samples show a similar behaviour, demonstrating a "universal" temperature dependence with NW length.

In our lightly doped NWs, impurity scattering of phonons is negligible and cannot suppress phonon drag. This leaves surface scattering due to restricted NW dimensions and surface roughness as the likely suppression mechanism. As $l_{p} \sim 1 \mu \mathrm{m}$ in lightly doped ${ }^{10}$ bulk $\mathrm{Si}$ at $300 \mathrm{~K}$ and increases at lower temperatures (Fig. 3(a) inset), $l_{p}$ is always greater than NW diameter. Strong surface scattering of phonons in the NWs then limits $l_{p} \sim \mathrm{NW}$ diameter and 
(a)

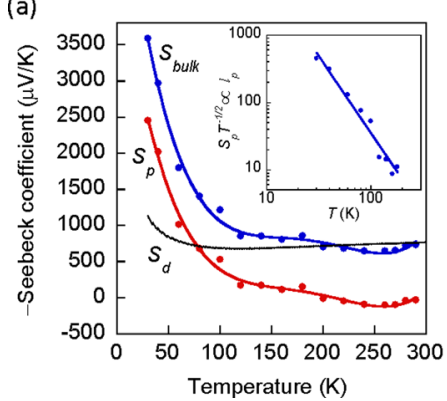

(b)

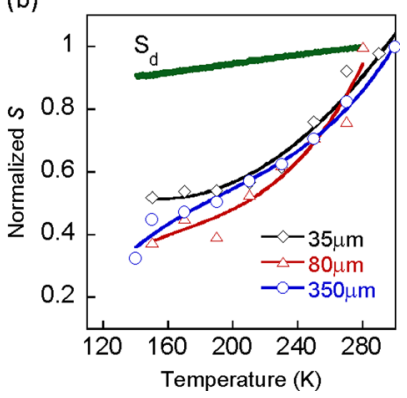

FIG. 3. (a) $S_{\text {bulk }}$ (measured), $S_{d}$ (calculated) and $S_{p}$ (extracted), vs. $T$ in bulk $\mathrm{Si}$. The inset shows $S_{p} T^{-1 / 2} \propto l_{p}$, the phonon mean free path, vs. $T$. (b) $S_{N W}$ normalized with the room-temperature values for three samples, vs. T. $S_{d}$ is shown for comparison.

suppresses $S_{p}$. This is the same underlying mechanism for reduced $\kappa$ in SiNWs, ${ }^{3,23}$ implying interdependence between $S_{N W}$ and $\kappa$. We also observe maximum $S_{N W} \sim 3 S_{\text {bulk }}$ at room temperature (Fig. 2(d)). This may be attributed to either a change in $r$ (Eq. (1)) due to the change in scattering mechanism from phonon to surface scattering or an increase in $E_{C}-E_{F}$ due to surface effects. Finally, we estimate $Z T$ in the NWs, at $300 \mathrm{~K}$. Using the maximum $S_{N W}=2300 \mu \mathrm{V} / \mathrm{K}$, $\kappa_{N W}=8 \mathrm{~W} / \mathrm{m} \mathrm{K}$ (Ref. 3), and assuming $\sigma$ in the NWs is unchanged from bulk, ${ }^{24}$ we find $Z T_{N W}=0.005 \approx 150 Z T_{\text {bulk }}$. This suggests a large improvement in the TE properties of lightly doped SiNWs compared to bulk Si.

The authors would like to acknowledge M. Green, K. Fobelets, C. Li, and V. Stevens for useful discussions and the financial support of the E.ON International Research Initiative.

\footnotetext{
${ }^{1}$ A. Majumdar, Science 303, 777-778 (2004).

${ }^{2}$ F. DiSalvo, Science 285, 703-706 (1999).
}

${ }^{3}$ A. I. Hochbaum, R. Chen, R. D. Delgado, W. Liang, E. C. Garnett, M. Najarian, A. Majumdar, and P. Yang, Nature 451, 163-167 (2008).

${ }^{4}$ A. Boukai, Y. Bunimovich, J. Tahir-Kheli, J. K. Yu, W. Goddard, and J. Heath, Nature 451, 168-171 (2008).

${ }^{5}$ J. R. Drabble and H. J. Goldsmid, Thermal Conduction in Semiconductors (Pergamon Press, Oxford, 1961).

${ }^{6}$ G. J. Snyder and E. S. Toberer, Nature Mater. 7, 105-114 (2008).

${ }^{7}$ M. S. Dresselhaus, G. Chen, M. Y. Tang, R. G. Yang, H. Lee, D. Z. Wang, Z. F. Ren, J.-P. Fleurial, and P. Gogna, Adv. Mater. 19, 1043-1053 (2007).

${ }^{8}$ M. S. Dresselhaus, G. Dresselhaus, X. Sun, Z. Zhang, S. B. Cronin, and T. Koga, Phys. Solid State 41, 679-682 (1999).

${ }^{9}$ S. K. Bux, J. P. Fleurial, and R. B. Kaner, Chem. Commun. 46, 8311-8324 (2010).

${ }^{10}$ L. Weber and E. Gmelin, Appl. Phys. A 53, 136-140 (1991).

${ }^{11}$ K. Q. Peng, Y. J. Yan, S. P. Gao, and J. Zhu, Adv. Mater. 16, 1164-1167 (2002).

${ }^{12}$ Z. Huang, N. Geyer, P. Werner, J. de Boor, and U. Gosele, Adv. Mater. 23, 285-308 (2011).

${ }^{13}$ Y. Qu, L. Liao, Y. Li, H. Zhang, and Y. Huang, Nano Lett. 9, 4539-4543 (2009).

${ }^{14}$ H. Fang, Y. Wu, J. Zhao, and J. Zhu, Nanotechnology 17, 3768-3774 (2006).

${ }^{15}$ T. M. Tritt, Mat. Res. Soc. 478, 25-36 (1997).

${ }^{16}$ T. M. Tritt and V. Browning, Semicond. Semimetals 69, 25-49 (2001).

${ }^{17}$ Thermoelectrics Handbook: Macro to Nano, edited by D. M. Rowe (CRC Press, 2006).

${ }^{18}$ G. K. White and S. J. Collocott, J. Phys. Chem. Ref. Data 13, 1251-1257 (1984).

${ }^{19}$ C. Kittel, Introduction to Solid State Physics (John Wiley \& Sons, New York, 1976).

${ }^{20}$ G. S. Nolas, J. Sharp, and J. Goldsmid, Thermoelectrics Basic Principles and New Materials Developments (Springer, Berlin, 2001).

${ }^{21}$ H. J. Goldsmid, Applications of Thermoelectricity (Methuen \& Co., London, 1960).

${ }^{22}$ S. M. Sze, Physics of Semiconductor Devices (John Wiley \& Sons, New York, 1981).

${ }^{23}$ Z. Wang, Z. Ni, M. Chen, K. Bi, and Y. Chen, Physica B 406, 2515-2520 (2011).

${ }^{24}$ J. Jie, W. Zhang, K. Peng, G. Yuan, C. S. Lee, and S.-T. Lee, Adv. Funct. Mater. 18, 3251-3257 (2008). 